\title{
Histone deacetylases 1 and 2 act in concert to promote the G1-to-S progression
}

\author{
Teppei Yamaguchi, ${ }^{1,4}$ Fabien Cubizolles, ${ }^{4}$ Yu Zhang, ${ }^{2}$ Nina Reichert, Hubertus Kohler, \\ Christian Seiser, ${ }^{3}$ and Patrick Matthias ${ }^{5}$ \\ Friedrich Miescher Institute for Biomedical Research, Novartis Research Foundation, 4058 Basel, Switzerland
}

\begin{abstract}
Histone deacetylases (HDACs) regulate gene expression by deacetylating histones and also modulate the acetylation of a number of nonhistone proteins, thus impinging on various cellular processes. Here, we analyzed the major class I enzymes HDAC1 and HDAC2 in primary mouse fibroblasts and in the B-cell lineage. Fibroblasts lacking both enzymes fail to proliferate in culture and exhibit a strong cell cycle block in the G1 phase that is associated with up-regulation of the CDK inhibitors $\mathrm{p} 21^{\mathrm{WAF} 1 / \mathrm{CIP1}}$ and $\mathrm{p} 57^{\mathrm{Kip} 2}$ and of the corresponding mRNAs. This regulation is direct, as in wild-type cells HDAC1 and HDAC2 are bound to the promoter regions of the p21 and p57 genes. Furthermore, analysis of the transcriptome and of histone modifications in mutant cells demonstrated that HDAC1 and HDAC2 have only partly overlapping roles. Next, we eliminated HDAC1 and HDAC2 in the B cells of conditionally targeted mice. We found that B-cell development strictly requires the presence of at least one of these enzymes: When both enzymes are ablated, B-cell development is blocked at an early stage, and the rare remaining pre-B cells show a block in G1 accompanied by the induction of apoptosis. In contrast, elimination of HDAC1 and HDAC2 in mature resting B cells has no negative impact, unless these cells are induced to proliferate. These results indicate that HDAC1 and HDAC2, by normally repressing the expression of p21 and p57, regulate the G1-to-S-phase transition of the cell cycle.
\end{abstract}

[Keywords: Histone deacetylases; cell cycle control; p21; p57; B-cell development]

Supplemental material is available at http://www.genesdev.org.

Received August 5, 2009; revised version accepted December 24, 2009.

Covalent modifications of core histones were linked to transcriptional activity nearly half a century ago (Allfrey et al. 1964), yet the details of the molecular mechanisms involved were unexplored until recently. Acetylation on histone lysine residues is positively correlated with gene transcription, and genome-wide studies showed that the bulk of histone $\mathrm{H} 3$ and $\mathrm{H} 4$ acetylation is enriched in gene active loci such as euchromatic regions, whereas hypoacetylated histones are detected in gene-repressed loci such as heterochromatic regions, centromeres, and telomeres (Vogelauer et al. 2000; Kurdistani et al. 2004; Roh et al. 2005). Histone acetylation occurs reversibly at the $\varepsilon$-amino group of lysine residues in all core histones, mainly at the tails but also at a few residues within the globular domain (Berger 2007). Histone acetylation is

Present addresses: ${ }^{1}$ Department of Molecular and Cell Biology, University of California at Berkeley, 16 Barker Hall \#3204, Berkeley, CA 94720 USA; ${ }^{2}$ The CBR Institute for Biomedical Research, Harvard Medical School, Boston, MA 02115, USA; ${ }^{3}$ Department of Medical Biochemistry, Max F. Perutz Laboratories, Medical University of Vienna, Vienna Biocenter, Room 2630, Dr. Bohr-Gasse 9/2, A-1030 Vienna, Austria.

${ }^{4}$ These authors contributed equally to this work.

${ }^{5}$ Corresponding author.

E-MAIL patrick.matthias@fmi.ch; FAX 061-697-39-76.

Article is online at http://www.genesdev.org/cgi/doi/10.1101/gad.552310. proposed to regulate gene expression by making the chromatin structure open and also by providing a docking site for bromodomain-containing proteins, which facilitates the binding of transcription factors to initiate and enhance transcription (Berger 2007).

This reversible acetylation is mediated by two opposing enzymatic activities: histone acetyltransferases (HATs) and histone deacetylases (HDACs). HDACs catalyze the deacetylation of lysine residues of histones as well as of an increasing number of other proteins, such as p53 (Luo et al. 2000), HSP90 (Bali et al. 2005; Kovacs et al. 2005), or $\alpha$-tubulin (Hubbert et al. 2002; Zhang et al. 2003, 2008; for review, see Minucci and Pelicci 2006). In mammals, 18 HDACs that can be grouped into four classes have been identified. The class I enzymes HDAC1 and HDAC2 are the closest homologs of yeast Rpd3, which is the main HDAC regulating the global level of histone acetylation in yeast (Kurdistani et al. 2002). In mammals, HDAC1 and HDAC2 are highly related proteins showing $87 \%$ amino acid identity in mice; they are predominantly localized in the nucleus and are coexpressed in a wide range of tissues and cell types. HDAC1 and HDAC2 have been identified as components of three distinct repressive complexes: the Sin3, NuRD, and Co-REST complexes, which, through interaction with a number of 
DNA-binding transcription factors, are recruited to many genes (Yang and Seto 2008).

Gene targeting studies of HDAC1 have demonstrated that this protein is essential for embryogenesis (Lagger et al. 2002). Furthermore, HDAC1-null embryonic stem (ES) cells exhibit a proliferation defect, in part caused by up-regulation of the cell cycle inhibitor p21, which was found to be a target of HDAC1 in these cells (Lagger et al. 2003). Recently, the p21 promoter was shown to be a common target of HDAC1 and HDAC2, at least in cancer cells (Lin et al. 2008). In contrast, HDAC2deficient mice-made recently following a slightly different inactivation strategy-show partial or complete perinatal lethality, depending on the allele (Montgomery et al. 2007; Trivedi et al. 2007; Zimmermann et al. 2007; Guan et al. 2009). Conditional deletion was used to test the role of HDAC1 or HDAC2 in cardiomyocytes and in neuronal precursors; in both cases, normal development was observed. However, combined loss of the two proteins was found to lead to severe defects in cell survival and development, suggesting functional redundancy between HDAC1 and HDAC2 in these biological systems (Montgomery et al. 2007, 2009). In spite of these recent studies, the physiological functions of HDAC1 and HDAC2 have remained largely unaddressed, and the molecular mechanisms by which these enzymes regulate cellular function are not well understood.

Here we examined the role of HDAC1 and HDAC2 in mouse embryonic fibroblasts (MEFs) and in B cells. MEFs lacking only one of these two proteins show moderate or no proliferation defects, whereas the combined loss of both proteins completely blocks cell cycle progression in the G1 phase and is associated with up-regulation of the cyclin-dependent kinase (CDK) inhibitors (CDKis) $\mathrm{p} 21^{\mathrm{WAF} 1 / \mathrm{CIP} 1}$ and $\mathrm{p} 57^{\mathrm{Kip} 2}$. Importantly, HDAC1 and HDAC2 bind to the promoter regions of the $p 21$ and p57 genes, indicating that they directly regulate their expression to control cell cycle progression. Down-regulation of p21 and p57 by shRNA in double-deficient cells largely alleviates the cell cycle block. Similar results were obtained in vivo by elimination of these enzymes in the B-cell lineage. Using different cre-expressing strains and mice conditionally targeted at the HDAC1 and HDAC2 loci, we eliminated these enzymes either in the entire B-cell lineage or specifically in nondividing terminally differentiated B cells. We found that, in the absence of either of these proteins, B-cell development proceeds normally. Simultaneous elimination of both enzymes in early B-cell progenitors leads to a dramatic block in B-cell development at the preB-cell stage, accompanied by G1 arrest and apoptosis induction. In contrast, mature B cells lacking both enzymes show no defect in cell viability; however, they fail to proliferate and undergo rapid apoptosis upon exogenous mitogenic stimulation. These results, obtained in different cellular systems, indicate that HDAC1 and HDAC2 act in concert to promote the G1-to-S-phase transition by inhibiting the expression of p21 and $p 57$.

\section{Results \\ Conditional inactivation of HDAC1 and HDAC2 in primary mouse fibroblasts}

The HDAC1 and HDAC2 proteins are highly homologous and share $87 \%$ identity at the amino acid level. Although the initial germline knockout (KO) of HDAC1 in mice was described to be embryonic-lethal (Lagger et al. 2002), recent results have shown that deletion of HDAC1 in adult tissues has no obvious phenotypic consequences (Montgomery et al. 2007, 2009), suggesting that another HDAC could redundantly replace HDAC1. Given the high homology between HDAC1 and HDAC2, and previous results showing that the HDAC2 protein is up-regulated in HDAC1-deficient ES cells (Lagger et al. 2002), HDAC2 appears to be a good candidate. To directly test this, we set out to analyze the functions of these two enzymes in MEFs. For this, we designed a system allowing us to down-regulate in an inducible manner HDAC1 and/or HDAC2 in the same parental fibroblast cell line. To do so, we combined cre-loxP recombination of the HDAC1 gene with RNAi to down-regulate the product of HDAC2 (Fig. 1A). First, mice conditionally targeted at the HDAC1 locus were generated (see the Materials and Methods; Supplemental Fig. 1A). Next, MEFs were prepared from individual $H D A C 1^{F / F}$ embryos and were transduced with two different retroviruses: One virus expresses the tamoxifen-inducible cre recombinase Cre-ERT2 (Indra et al. 1999) and green fluorescent protein (GFP), and the second virus expresses either a small hairpin microRNA (miRNA) against HDAC2 or a scrambled version thereof together with a puromycin resistance gene (Fig. 1A). Selecting cells that are puromycin-resistant and $\mathrm{GFP}^{+}$allowed us to generate cell pools that were infected with both viruses. $H D A C 1^{F / F}$ MEFs expressing either a scrambled miRNA or a miRNA against HDAC2 can be induced by addition of tamoxifen to delete HDAC1, thereby generating four different genotypes: wild-type, HDAC1 KO, HDAC2 knockdown (Kd), and HDAC1/2 $\mathrm{KO} / \mathrm{Kd}$.

In both wild-type and HDAC2 $\mathrm{Kd}$ cells, addition of tamoxifen induces complete recombination of the flox allele, as measured by a PCR assay (Supplemental Fig. 1B, cf. lanes 1,2 and lanes 3,4). The content of HDAC1 and HDAC2 proteins in these different cells was then analyzed by Western blot. To accurately quantify the level of proteins, we used a fluorochrome-coupled secondary antibody and quantified the blots with an infrared imaging system. In the HDAC2 Kd cells, only $1 \%-2 \%$ of the protein remained (Fig. 1B, lanes 3,4). After tamoxifen addition, no HDAC1 protein was detectable, suggesting a complete recombination, as seen at the DNA level by PCR analysis (Fig. 1B, lanes 2,4). In HDAC1 KO cells, we observed an increase of HDAC2 content by $~ 50 \%$ (Fig. 1B, cf. lanes 1 and 2). An up-regulation of HDAC2 protein in the absence of HDAC1 was also seen in ES cells (Lagger et al. 2002). The opposite is also true, and, in the absence of HDAC2, the HDAC1 level increases about three times (Fig. 1B, cf. lanes 1 and 3). 
A

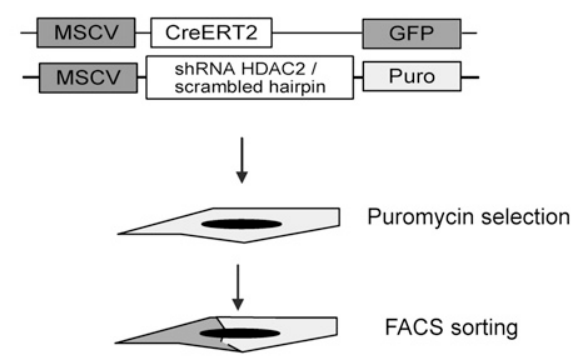

Stable cell line expressing CreERT2 + ShRNA
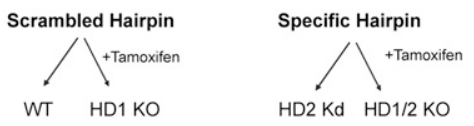

B

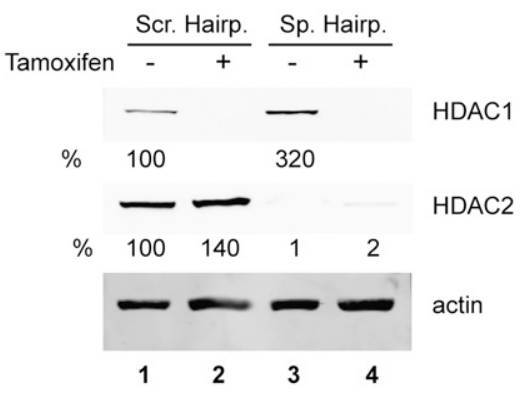

C

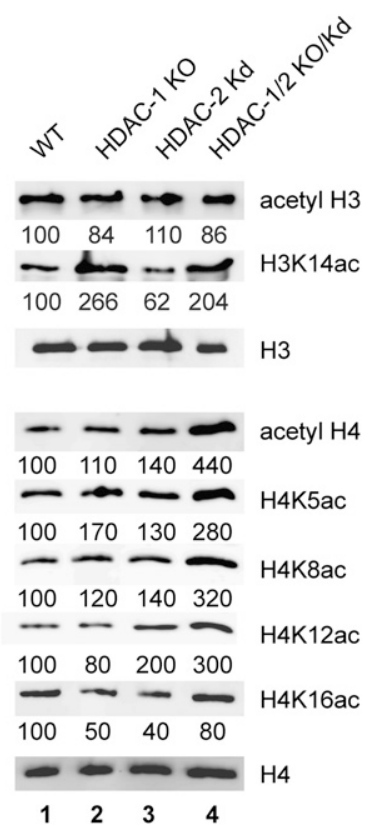

Figure 1. Elevated histone acetylation in MEFs lacking HDAC1 or HDAC2. (A) Experimental strategy. Primary MEFs from $H D A C 1^{F / F}$ mice were infected with two different retroviruses: One virus expresses CreERT2 and GFP, and the second virus encodes puromycin resistance and also expresses either a small hairpin miRNA against HDAC2 or a scrambled small hairpin miRNA. Following infection, stable cell pools are obtained by puromycin selection followed by FACS sorting of GFP-positive cells. Addition of tamoxifen allows us to generate the four different genotypes (wild-type [WT], HDAC1 $\mathrm{KO}, \mathrm{HDAC} 2 \mathrm{Kd}$, and $\mathrm{HDAC} 1 / 2 \mathrm{KO} / \mathrm{Kd}$ ) from the same starting cells, allowing a direct comparison. (B) Protein extracts were prepared from cells of the four different genotypes and probed with antibodies against HDAC1, HDAC2, and actin, as indicated. Western blot analysis was done using the Odyssey Infrared Imaging System. The quantification of band intensities is indicated below using arbitrary units. (Lane 1) The amount of HDAC1 or HDAC2 in the initial cells was set to $100 \%$. (C) Loss of HDAC1 and/or HDAC2 leads to selective increase in histone acetylation. Nuclear extracts from the indicated MEF cells were probed with antibodies recognizing $\mathrm{H} 3$ or $\mathrm{H} 4$, or the acetylated forms of $\mathrm{H} 3, \mathrm{H} 4, \mathrm{H} 3 \mathrm{~K} 14, \mathrm{H} 4 \mathrm{~K} 5$, $\mathrm{H} 4 \mathrm{~K} 8, \mathrm{H} 4 \mathrm{~K} 12$, or H4K16. The signal for H3 and $\mathrm{H} 4$ is presented, demonstrating equal protein loading. Western blot analysis was done using the Odyssey Infrared Imaging System. The quantification of band intensities is indicated below using arbitrary units. (Lane 1) The amount in the wild-type cells was set to 100, and all values were corrected for the $\mathrm{H} 3$ or $\mathrm{H} 4$ signal. A representative experiment is presented.

\section{Elevated histone acetylation in double-mutant MEFs}

We examined the effect of HDAC inactivation on histone acetylation, since both HDACs are known to be localized exclusively in the nucleus (as shown in Supplemental Fig. 2A; Rountree et al. 2000; Taplick et al. 2001). For this, histones were extracted from the different MEFs, and the level of histone acetylation was visualized by Western blotting with specific antibodies (Fig. 1C). The acetylation level of the bulk histone $\mathrm{H} 3$ did not show significant differences between wild-type or mutant cells, and this was also true for specific residues such as H3K9 or H3K4 (data not shown). In contrast, acetylation on H3K14 showed an $\sim 2.5$-fold increase when HDACl is deleted. This effect appears to be specific to $\mathrm{HDAC} 1$, as it was not observed in HDAC2 $\mathrm{Kd}$ cells, and $\mathrm{KO} / \mathrm{Kd}$ cells did not show further enhanced H3K14 acetylation compared with HDAC1 KO cells. Unlike histone $\mathrm{H} 3$, bulk histone $\mathrm{H} 4$ showed acetylation changes between the different cellular backgrounds. Indeed, single-mutant cells showed a slight increase (1.1-fold to 1.4-fold), while double-mutant cells had a more than fourfold increase in total $\mathrm{H} 4$ acetylation. This effect was also observed by immunofluorescence: In $\mathrm{KO} / \mathrm{Kd}$ cells, the intensity of acetylation increased much more than in wild-type cells, and was present throughout the nucleus (Supplemental Fig. 2B). Similar observations were made for the acetylation level of individual residues such as $\mathrm{H} 4 \mathrm{~K} 5 \mathrm{ac}, \mathrm{H} 4 \mathrm{~K} 8 \mathrm{ac}$, or $\mathrm{H} 4 \mathrm{~K} 12 \mathrm{ac}$ : In these cases, acetylation was moderately enhanced in the individual mutant cells and more than additively increased in the double-mutant cells to reach more than threefold increase in the case of H4K8. In contrast, acetylation of $\mathrm{H} 4 \mathrm{~K} 16$ was slightly reduced in all mutant cells. Together, these observations suggest that HDAC1 and HDAC2 may have different substrate specificities on histone residues. 


\section{HDAC1/2 double-mutant MEFs cease proliferation and undergo apoptosis}

The proliferation potential of the different MEF lines was monitored by counting the number of viable cells over time. We did not observe significant proliferation differences between wild-type and HDAC2 Kd cells (Fig. 2A, cf. black line and gray line). Furthermore, similar treatment of wild-type cells with tamoxifen did not impair their proliferation (see Supplemental Fig. 3A). In contrast, when we induced the deletion of HDAC1 in $H D A C 1^{F / F}$ cells, we observed a slow proliferation phenotype. A similar phenotype had been described previously for ES cells lacking HDAC1 (Lagger et al. 2002). Strikingly, the double-mutant MEFs completely stopped proliferating (Fig. 2A, dashed gray line) following $4 \mathrm{~d}$ of tamoxifen

A

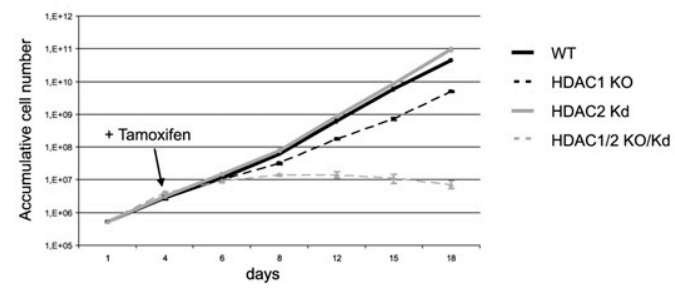

B

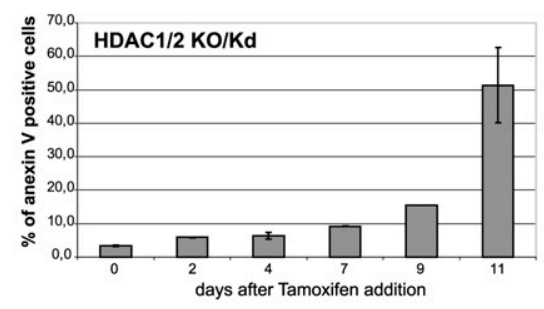

C

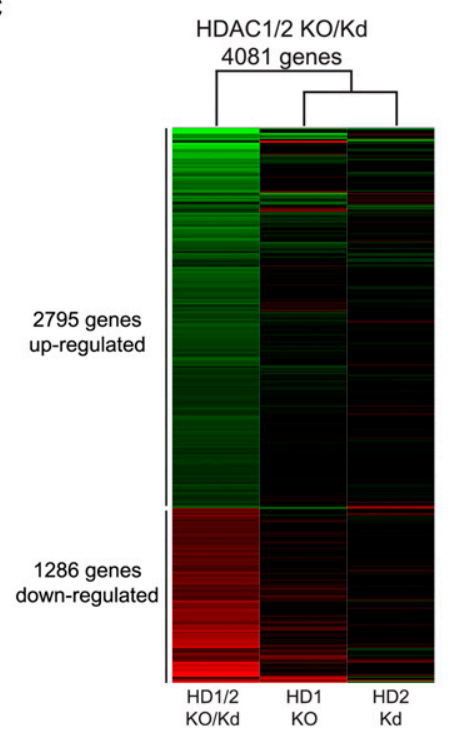

D

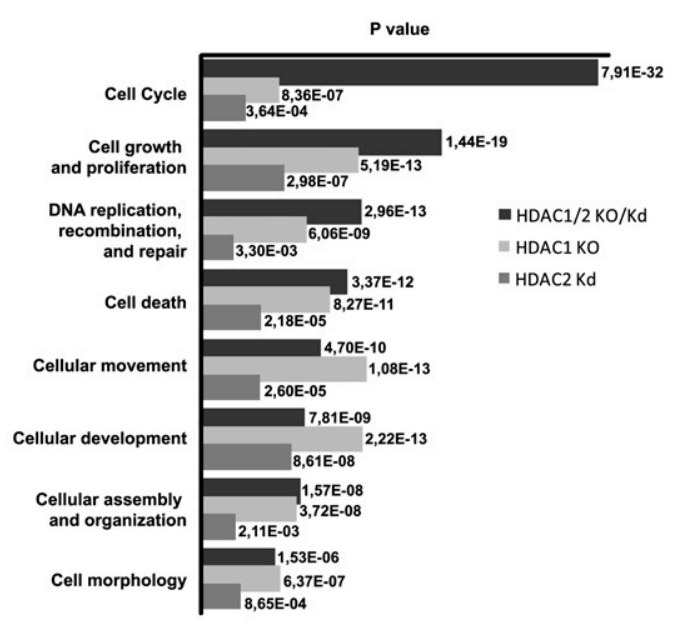

Figure 2. Elimination of HDAC1 and HDAC2 leads to impaired cell proliferation and induction of apoptosis. $(A)$ Growth curve. MEFs of the different genotypes were passaged every 3 or $4 \mathrm{~d}$, the total number of cells was counted, and cells were reseeded at $5 \times 10^{5}$ cells per 100-mm dish. Tamoxifen was added at day 4. Each growth curve is the average ( \pm SD) of three independent MEF cells generated from different embryos. (Solid black line) Wild-type cells; (black dashed line) HDAC1 KO cells; (solid gray line) HDAC2 Kd cells; (gray dashed line) HDAC1/2 KO/Kd cells. (B) Analysis of apoptosis by annexin V staining. HDAC1 ${ }^{F / F}$ MEF cells expressing Cre-ERT2 and a small hairpin miRNA against HDAC2 were followed over time after addition of tamoxifen. After fixation and staining with an annexin $\mathrm{V}$ antibody, the cells were analyzed by flow cytometry. The bar graph represents the average of three independent experiments $( \pm S D) .(C)$ Gene expression profiles. Supervised hierarchical clustering was performed using comparative expression values of the gene lists indicated above the dendrogram (for gene lists, see Supplemental Table 1). In all cases, the expression value for the wild-type cells was used to normalize expression levels. $(D)$ Gene Ontology term analysis of the main gene expression changes observed in the different MEF lines. The networks and molecular functions scoring the eight highest $P$-value in $\mathrm{HDACl} / 2 \mathrm{KO} / \mathrm{Kd}$ cells are listed together with the $P$-value in each single-mutant cell. 
treatment, a time at which the HDAC1 protein is no longer detectable by Western blot (see Fig. 3D). After the cells stop proliferating, most of them remain viable for $>1 \mathrm{wk}$, with gradual detachment from the dish followed by cell death. These double-mutant cells were tested by annexin $\mathrm{V}$ staining at different time points for the appearance of apoptosis. As shown in Figure 2B, the percentage of apoptotic cells gradually increased following tamoxifen addition to reach $\sim 15 \%$ of the cells at day 9 and $\sim 50 \%$ at day 11 . Apoptosis was also examined in the other MEF lines after identical treatment with tamoxifen or vehicle. In this case, however, we did not observe more apoptosis in HDAC1 KO or HDAC2 Kd cells than in wild-type cells (Supplemental Fig. 4A).

Next we carried out a transcriptome analysis to identify the genes deregulated upon loss of HDAC1, HDAC2, or both. For this, RNA was isolated from each MEF line and subjected to a microarray analysis using the Affymetrix GeneArray MOE430 version 2.0. We found that the number of deregulated genes in each cell line was correlated with the severity of the proliferation phenotype; the least changes were observed in HDAC2 Kd cells (193 genes up-regulated and 90 genes down-regulated) (Supplemental Fig. 5A), and the most changes were seen in $\mathrm{HDAC1} / 2 \mathrm{KO} / \mathrm{Kd}$ cells (2795 genes up-regulated and 1286 genes down-regulated) (Fig. 2C). HDAC1 KO cells showed an intermediate level of deregulation (412 upregulated genes and 301 down-regulated genes) (Supplemental Fig. 5A). The number of deregulated genes in double-mutant cells was much greater than the combined numbers of HDAC1 KO and HDAC2 Kd cells. This more than additive effect in double-mutant cells suggests that both proteins could redundantly regulate the expression of many genes. It may also reflect some phenotypic consequences of the proliferation stop observed with HDAC1/2 KO/Kd cells. We compared the overlap of deregulated genes in each genotype and found that only a small fraction of the genes were commonly deregulated
A

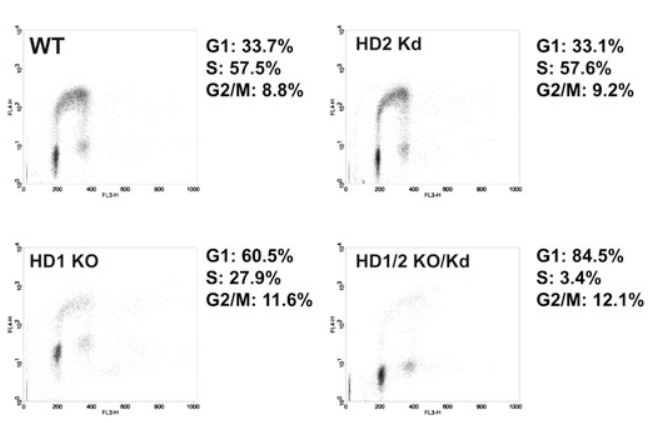

C

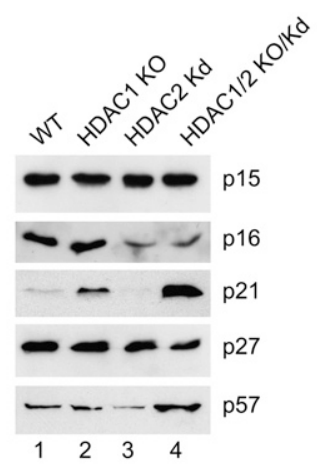

D
B
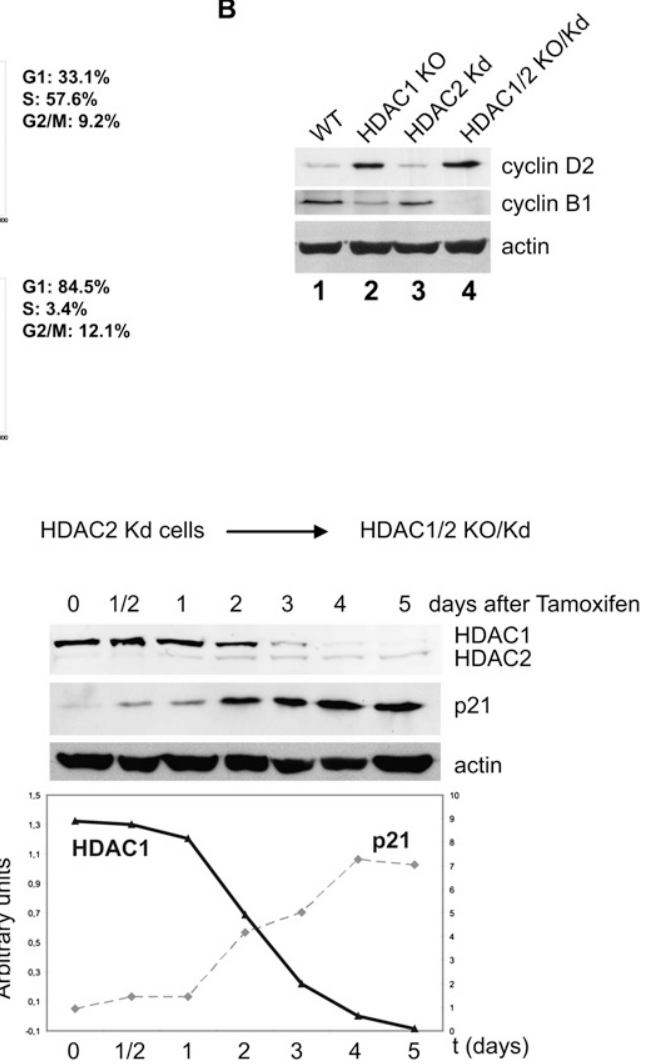

Figure 3. Loss of HDAC1 and HDAC2 leads to a cell cycle block in G1 accompanied by increased levels of CDKis p21 ${ }^{\text {WAF1/CIP1 }}$ and p5 $7^{\text {Kip2 }}$. (A) Cell cycle analysis by flow cytometry. Following a 30-min pulse of BrdU, cells were collected and ethanol-fixed. After staining with an antibody against BrdU and 7-AAD, cells were analyzed by FACS. Representative dot plots are shown for each genotype, and the percentage of cells in the different cell cycle phases are indicated to the right of each panel. $(B)$ Protein extracts were prepared from wild-type, HDAC1 KO, HDAC2 $\mathrm{Kd}$, and $\mathrm{HDAC} 1 / 2 \mathrm{KO} / \mathrm{Kd}$ cells and probed with antibodies against cyclin D2, cyclin B1, and actin, as indicated. $(C)$ Deregulated expression of CDKis in the absence of HDAC1 and HDAC2. Protein extracts were prepared from wild-type, HDAC1 KO, HDAC2 Kd, and HDAC1/2 KO cells and probed with antibodies against p15 (cdkn2b), p16 (cdkn2a), p21 (cdkn1a), p27 (cdkn1b), and p57 (cdkn1c), as indicated. (D) p21 protein expression increases proportionally to the down-regulation of HDAC1. Deletion of HDAC1 was induced in HDAC2 Kd cells by addition of tamoxifen, and the level of HDAC1, HDAC2, and p21 proteins was followed by Western blot analysis at different time points, as indicated. Band intensities were quantified using the Odyssey Infrared Imaging System and were plotted using arbitrary units. 
between HDAC1 and HDAC2 mutant cells (Supplemental Fig. 5B). One-hundred genes are common in HDAC1 $\mathrm{KO}$ and HDAC2 Kd cells, which are $14 \%$ of the genes deregulated in $\mathrm{HDAC} 1 \mathrm{KO}$ and $35 \%$ of the genes deregulated in HDAC2 Kd cells. The gene expression changes were analyzed for networks and molecular functions through the use of Ingenuity Pathways Analysis (Ingenuity Systems) (Fig. 2D). In good agreement with the proliferation defect observed with $\mathrm{HDACl} / 2 \mathrm{KO} / \mathrm{Kd}$ cells (Fig. 2A), the most deregulated genes were identified as components of molecular networks for "cell cycle" and "cell growth and proliferation," with a very high $P$-value compared with the other networks (Fig. 2D). Deregulation of genes belonging to these two categories were also observed in transcriptomic studies done in $H D A C 1^{-/-} \mathrm{ES}$ cells (Zupkovitz et al. 2006) or in HDACl/2 Kd human cancer cells (Senese et al. 2007).

\section{HDAC1/2 double-mutant cells are blocked} in $G 1$ and accumulate CDKis

As HDAC1/2 double-mutant MEFs stopped dividing, and genes involved in the cell cycle are overrepresented in the genome-wide expression profile, we next sought to identify molecular mechanisms underlying the proliferation defect. For this, 5-bromo-2-deoxyuridine (BrdU) incorporation assays were performed with cells in culture. As shown in Figure 3A, there were no cell cycle differences between wild-type and HDAC2 Kd cells ( $\sim 57 \%$ of cells in S phase and $\sim 33 \%$ in G1 for both genotypes), in good agreement with the growth curve experiments (Fig. 2A). In contrast, $\mathrm{HDAC} 1 \mathrm{KO}$ cells showed a strongly reduced percentage of cells in S phase $(27.9 \%)$ and a corresponding increase in G1 $(60.5 \%)$ (Fig. 3A, bottom left panel); this observation correlates well with the slow proliferation phenotype observed in the absence of HDAC1. Finally, the double-mutant $\mathrm{HDACl} / 2 \mathrm{KO} / \mathrm{Kd}$ MEFs have almost no cells in $S$ phase $(\sim 3 \%)$, and the vast majority of the cells $(84.5 \%)$ are arrested in G1 (Fig. 3A).

We next examined cell cycle regulators and investigated the cyclin content of the different MEFs. For this, cell extracts were probed for an early G1 cyclin (cyclin D2) and for a mitotic cyclin (cyclin B1). As presented in Figure 3B, HDAC1 KO MEFs show an accumulation of cyclin D2 and a reduced amount of cyclin B1 relative to wild-type cells (cf. lanes 1 and 2). In contrast, $\mathrm{HDACl} / 2$ $\mathrm{KO} / \mathrm{Kd}$ cells exhibit a stronger phenotype. Indeed, these cells present the same accumulation of cyclin D2, but are completely depleted of cyclin B1 (Fig. 3B, cf. lanes 1 and 4). Furthermore, the genome-wide gene expression profile showed a decrease of all cyclins except cyclin D family (E, A, and B) (see Supplemental Table 1). These results strongly suggest that the double-mutant cells are blocked in early G1, likely before the restriction point. Moreover, in MEFs lacking only HDACl, we observed a slow proliferation phenotype that may reflect impaired progression through G1. The phenotype obtained in these mutant cells suggests that it could be due to a misregulation of some small CDKis. We therefore examined the content of several of the CDKis by Western blotting. As shown in Figure 3C, p21 is up-regulated in HDAC1 KO $\mathrm{MEFs}$, and the increase is even larger in double-mutant cells (Fig. 3C, lanes 2,4), which also show an up-regulation of p57 (Fig. 3C, lane 4). This deregulation of p21 and p57 is due to the elimination of HDAC1 and HDAC2, as it is not observed in wild-type cells treated with tamoxifen (see Supplemental Fig. 3B).

In contrast, we did not observe any change in expression of the CDKis 27 or p15 (Fig. 3C). Finally, p16 was down-regulated in HDAC2 Kd cells, as well in the doublemutant cells, but was not affected by the absence of HDAC1 only. p21 and p57 are known to inhibit mainly CDK2/cyclinE (Malumbres et al. 2000). To exclude a possible artefact due to the use of RNAi, the same experiments were repeated using MEFs conditionally targeted for both genes $\left(H D A C 1 / 2^{F / F}\right)$. In these cells as well, deletion of HDAC1 and HDAC2 leads to G1 arrest and up-regulation of p21 and p57 (Supplemental Fig. 4B-D). Therefore, the strong accumulation of these two proteins in the double-mutant cells is the likely cause of the cell cycle block in G1 (see below). Next, we examined the disappearance of HDAC1 and the accumulation of $\mathrm{p} 21$ over time upon tamoxifen treatment of HDAC2 Kd cells (Fig. 3D). After $4 \mathrm{~d}$ of tamoxifen treatment, the HDAC1 protein is almost undetectable, and the cells become growth-arrested (Figs. 2A, 3D). At this time point, the level of p21 already reaches its plateau. The quantification of these Western blots (Fig. 3D, bottom panel) shows an inversely proportional relation between the amounts of $\mathrm{HDAC} 1$ and $\mathrm{p} 21$ proteins, strongly suggesting that HDAC1 could control p21 transcription directly.

p21 $1^{\text {WAF1/CIP1 }}$ and $p 57^{\text {Kip2 }}$ are two targets of HDAC1 and HDAC2 and are up-regulated in the double-mutant cells

We then performed chromatin immunoprecipitation (ChIP) experiments with either HDAC1 or HDAC2 to test the presence of these proteins on the $p 21$ and $p 57$ promoters. HDAC1 was immunoprecipitated in wildtype cells, in HDAC1 KO cells (control for immunoprecipitation), and in HDAC2 Kd cells where the HDAC1 protein is up-regulated. In parallel, HDAC2 was immunoprecipitated in the same cells, except that, in this case, the HDAC2 Kd sample represents the immunoprecipitation control. As shown in the left panel of Figure 4A, the HDAC1 immunoprecipitation with wild-type chromatin shows enrichment for both the $p 21$ and $p 57$ promoters, indicating that the HDACl protein is bound on both promoters in vivo. The enrichment is several-fold higher when $\mathrm{HDAC} 1$ is immunoprecipitated in chromatin from HDAC2 Kd cells. This result suggests that the up-regulation of HDAC1 protein seen in these cells leads to an increased loading on the $p 21$ and $p 57$ promoters. Similar results were obtained when $\mathrm{HDAC} 2$ was immunoprecipitated. Indeed, the protein is present on the $p 21$ and p57 promoters in wild-type cells (Fig. 4A, right panel), and a stronger enrichment was seen when HDAC2 is immunoprecipitated in the absence of HDAC1. Furthermore, expression of the $p 21$ RNA is elevated in double-mutant 
A

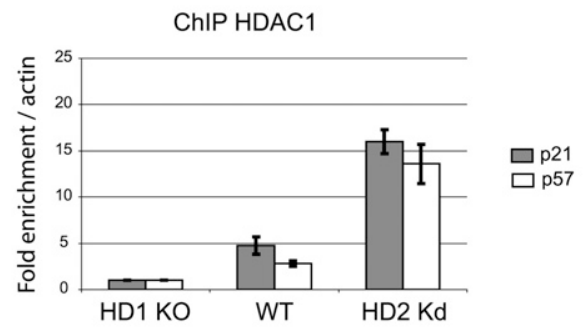

B

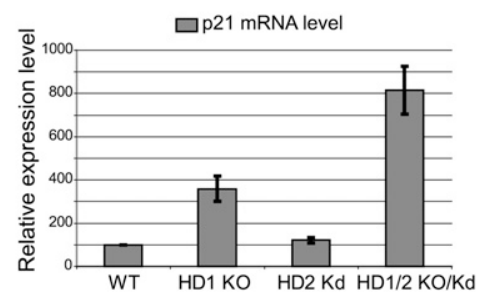

ChIP HDAC2
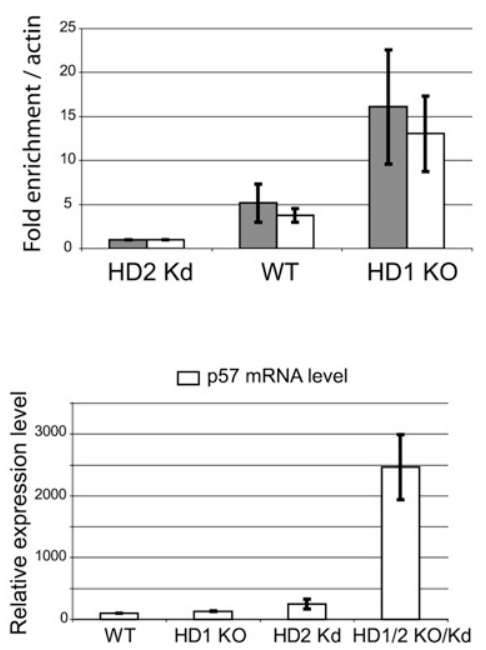

Figure 4. HDAC1 and HDAC2 are bound to the $p 21$ and $p 57$ promoters and regulate their expression. (A) Analysis of HDAC binding to the $p 21$ and $p 57$ promoters by ChIP assays. ChIP was performed for either HDAC1 or HDAC2 in wild-type, HDAC1 $\mathrm{KO}$, and HDAC2 $\mathrm{Kd}$ cells, as indicated. Enrichment was assessed by qPCR for the p21 and p57 promoters normalized to the amplification obtained with the actin promoter. Results presented are an average of three independent experiments $( \pm S D)$, with each PCR amplification performed in triplicate. $(B)$ Deregulated expression of the $p 21$ and $p 57$ genes in the absence of HDAC1 and HDAC2. RNA was extracted from the different cells as indicated, and was used for qRTPCR assays. The expression level of $p 21$ or p57 in wild-type cells was set to $100 \%$, after normalization by measuring the expression of Hprt1. The average $( \pm S D)$ from two independent experiments is presented. as well as in $H D A C 1^{-/-}$cells, and expression of the p57 RNA is elevated in double-mutant cells (Fig. 4B) in accordance with the genome-wide expression analysis (see Supplemental Table 1) and protein levels (Fig. 3C). A transcriptomic analysis of $H D A C 1^{-/-}$ES cells also had identified deregulation of the $p 21$ gene (Zupkovitz et al. 2006). Together, these results indicate that HDAC1 and HDAC2 directly negatively regulate the expression of the $p 21$ and $p 57$ genes, and thereby play a critical role for cell cycle progression through the G1 phase.

As mentioned above, HDAC1/2 $2^{F / F}$ MEFs /carrying a Cre-ERT2 vector) fully recapitulate the phenotype observed with the $\mathrm{HDAC} 1 / 2 \mathrm{KO} / \mathrm{Kd}$ cells: In the presence of tamoxifen, they up-regulate p21 and p57, are arrested in G1, and die by apoptosis, while in the absence of tamoxifen, they grow like wild-type cells. We therefore used these cells to attempt a rescue experiment. For this, $H D A C 1 / 2^{F / F}$ cells were infected with a lentivirus expressing an shRNA against GFP, or a mixture of lentiviruses expressing shRNAs against p21 and p57. Infected cells were either mock-treated or treated with tamoxifen to induce the deletion of HDAC1 and HDAC2, and, after $6 \mathrm{~d}$, a cell cycle analysis was done following BrdU incorporation. As shown in Supplemental Figure 6A, only $\sim 0.5 \%$ of the cells having deleted HDAC1 and HDAC2 and infected with an shRNA against GFP are in $S$ phase. In contrast, when the HDAC1/2 mutant cells express the shRNAs against p21 and p57 $\sim 6 \%$ are in $S$ phase. Quantitative RT-PCR (qRT-PCR) allowed us to assess the down-regulation of p21 and p57 expression (Supplemental Fig. 6B). Thus, down-regulation of p21 and p57 in HDAC1/2 mutant cells allows us to partially restore the cell cycle block.

B-cell-specific elimination of HDAC1 or HDAC2 does not impair B-cell development

We next wanted to see whether a similar phenotype could also be observed in vivo and ablated HDAC1 and HDAC2 in the B-cell lineage. In mammals, B lymphocytes are generated throughout the life of the animal, and the ontogeny of these cells has been well characterized. Mature B cells circulate and are found mostly in secondary lymphoid organs such as the spleen, while early developmental stages and progenitors are found in the bone marrow, where hematopoietic stem cells reside (for review, see Matthias and Rolink 2005). To be able to delete either HDAC1 or HDAC2, we generated a conditional null allele of $H D A C 2$ by the same strategy taken for the HDAC1 conditional mouse (Supplemental Fig. 1A).

The deletion of HDAC1 or HDAC2 was done by crossing $H D A C^{F / F}$ mice with mb1-cre transgenic mice in which cre expression is restricted to the B-cell compartment and is already robust in pre-BI cells, at the beginning of B-cell commitment (Fig. 5A; Hobeika et al. 2006). First, we analyzed the efficiency of flox allele deletion and examined by Western blotting the levels of HDAC1 and HDAC2 protein in splenic mature B cells of single-KO mice $\left(H D A C 1^{B \Delta / \Delta}\right.$ or $\left.H D A C 2^{B \Delta / \Delta}\right)$. As shown in Figure $5 \mathrm{~B}$, both proteins were below detection in these mice. Furthermore, no truncated forms of HDAC1 or HDAC2 were detected (data not shown). We also observed that the level of HDAC2 protein is up-regulated in the absence of HDAC1 (Fig. 5B, middle panel, cf. lanes 1 and 2). A PCR analysis revealed that no HDAC1 or HDAC2 flox alleles were detectable in mature B cells or in developing B cells as early as the pre-BII stage (data not shown). Together, these results confirm the successful inactivation of $\mathrm{HDAC} 1$ and HDAC2 throughout the B-cell lineage.

These mice were next analyzed by flow cytometry to determine the effect of ablating HDAC1 or HDAC2 in B cells. For this, a series of antibodies against specific cell surface markers was used to distinguish specific stages of B-cell development (Fig. 5A). We used antibodies against B220, a pan-B-cell marker, together with antibodies against CD19 or immunoglobulin (IgM) to identify committed B cells and distinguish between immature and 
A

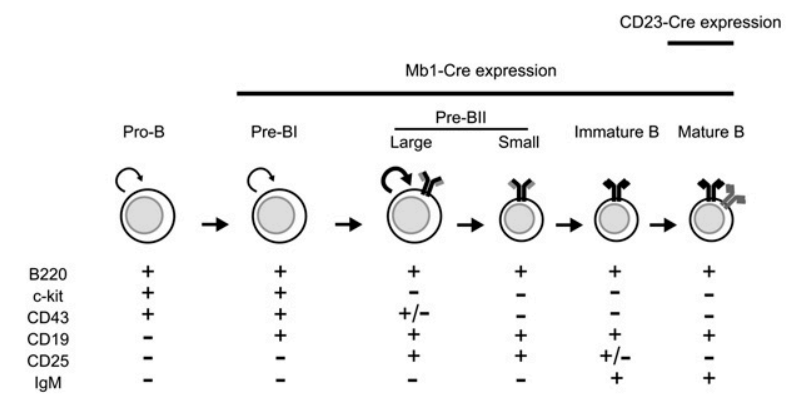

B

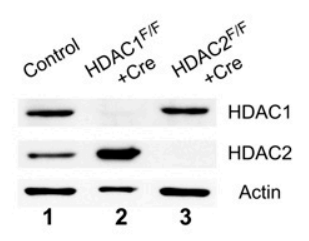

D

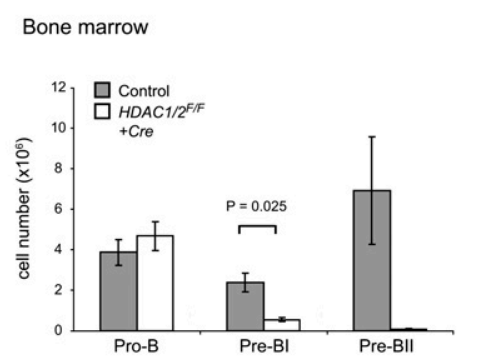

C

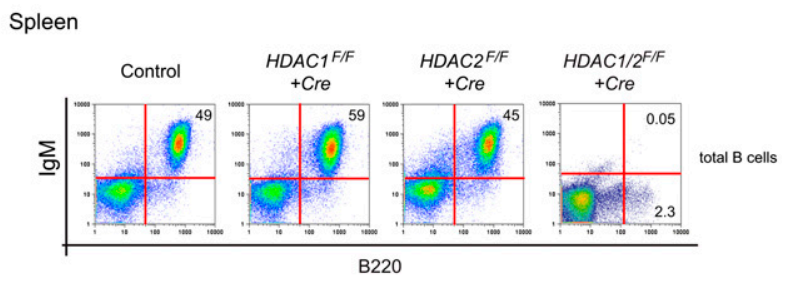

Bone marrow

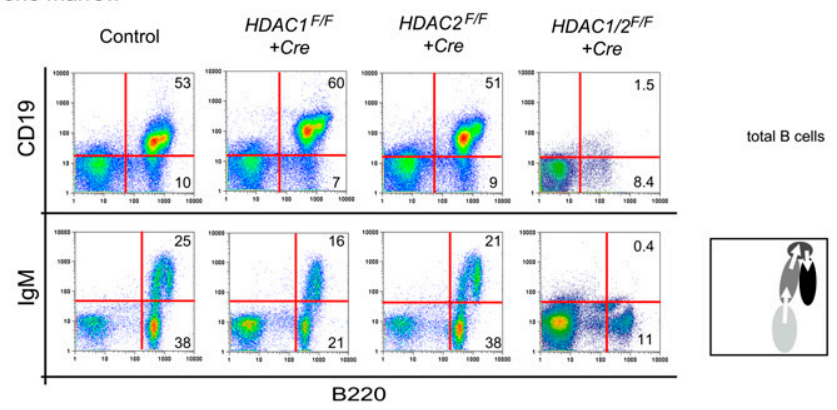

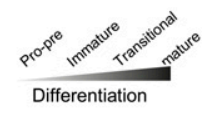

Figure 5. In vivo early B-cell development requires HDAC1 or HDAC2. (A) Scheme of early B-cell differentiation, depicting the different developmental stages and the expression of markers used to discriminate them: Marker expression is indicated by $(+)$, lack of expression is indicated by $(-)$. Curved arrows indicate relative cellular proliferation activities: very high in large pre-BII, intermediate in pro-BI and pre-BI, and low or absent in small pre-BII and immature and mature B cells. The expression of immunoglobulin (IgM) at the membrane is also depicted. At the top of the scheme, the pattern of cre expression in mb1-cre and CD23-cre mice is presented. (B) Western blot analysis of HDAC1 or HDAC2 in B cells of $H D A C 1^{F / F}$ or $H D A C 2^{F / F}+m b 1$-cre mice. Protein extracts from splenic mature $B$ cells were probed with antibodies against HDAC1, HDAC2, or actin, as indicated. $(C)$ Flow cytometric analysis of single-KO $\left(H D A C 1^{F / F}+m b 1\right.$ cre or $H D A C 2^{F / F}+m b 1$-crel or double-KO $\left(H D A C 1 / 2^{F / F}+\right.$ mb1-cre $)$ mice. Single-cell suspensions from spleen or bone marrow were stained with the indicated antibody combinations and analyzed by FACS. Individual dot plots are shown that are representative of more than three independent experiments. Numbers in plots represent the percentage of cells in the respective quadrant. A simplified scheme of the B-cell developmental progression based on the $\mathrm{B} 220$ and $\operatorname{IgM}$ markers ( $X$-axis/ $Y$-axis) is shown on the right. $(D)$ Total cell numbers present in different B-cell populations in the bone marrow. The absolute numbers were determined based on FACS analysis and cell counting. The histograms represent the mean \pm SE based on the analysis of at least four mice per genotype. mature B cells. As shown in Figure 5C, absence of HDAC1 or HDAC2 does not significantly impair the percentage of the B-cell populations present in the spleen or in the bone marrow (the slight differences in percentage between the different genotypes represent individual variations). Furthermore, cells at different stages of early B-cell development that were identified by markers such as c-kit, CD43, or CD25 were also present in normal proportions and absolute numbers in the bone marrow (data not shown). Thus, individually, HDAC1 and HDAC2 are dispensable for normal B-cell development.

\section{Deletion of HDAC1 and HDAC2 dramatically impairs $B$-cell differentiation}

The observations presented above suggest that HDAC1 and HDAC2 might be redundant or compensate each other in B cells, similar to the situation in MEFs; therefore, we eliminated both enzymes simultaneously. For this, mice were generated that were homozygously floxed at both genes and also carried the mb1-cre transgene $\left(H D A C 1 / 2^{F / F}+m b 1\right.$-cre $)$; littermates lacking the cre transgene were used as controls. As shown in Figure 5C, the spleen of mice lacking both HDAC1 and HDAC2 is virtually empty of circulating mature B cells that were identified using the pan-B-cell marker B220 in combination with IgM (Fig. 5C, top right panel). In the bone marrow, where B cells are generated, only very few B cells remain in $H D A C 1 / 2^{B \Delta / \Delta}$ mice (Fig. 5C, bottom panel). When B-cell differentiation was analyzed in detail, a block was identified as early as the pre-BI-cell stage. Quantification of absolute B-cell numbers in these animals shows that pre-BI cells are reduced by $60 \%-80 \%$, while at the 
pre-BII-cell stage, $<1 \%$ of the cells remained (Fig. 5D). Thus, normal progression through early B-cell development strictly requires the presence of HDAC1 or HDAC2.

\section{$B$ cells lacking HDAC1 and HDAC2 are blocked in G1 and undergo apoptosis}

Considering that pre-BII cells are highly proliferative (Rolink et al. 1993), and double inactivation of HDACs causes a strong G1 arrest in MEF cells, we examined the possibility that the developmental block observed in double-KO B cells reflects a function of HDAC1 and HDAC2 in cell cycle regulation. We therefore examined the cell cycle distribution of the pre-B cells in $H D A C 1 / 2^{F / F}+m b 1$-cre mice by in vivo BrdU incorporation assays. For this, BrdU was injected intraperitoneally to label cycling cells, and pre-BII cells were purified by fluorescence-activated cell sorting (FACS). Total DNA and incorporated BrdU were stained, and the cell cycle profile was determined by flow cytometry. Based on cell size, the pre-BII stage can be subdivided into two populations with distinct characteristics: large actively dividing cells that give rise to small resting cells that accumulate in high numbers. Here we focused our analysis on the large pre-BII cells. As shown in Figure $6 \mathrm{~A}, \sim 80 \%$ of the wild-type cells are in the S phase of the cell cycle, a reflection of the high proliferative index of the pre-BII population. In stark contrast, only $\sim 4.5 \%$ of the double-KO cells are in S phase, and $>87 \%$ are in the G1 phase. This G1 arrest coincides with the developmental block at the pre-BII stage described in Figure 5D. We also observed an increase of the sub-G1 population on the dot plot (Fig. 6A, right panel), likely reflecting apoptotic cells. To test whether these cells were apoptotic, we performed an annexin $\mathrm{V}$ staining followed by flow cytometric analysis. As is visible in Figure 6B (middle panel), at the large pre-BII stage, the majority of cells that are blocked in G1 are also positive for apoptosis. This proportion further increases at the small pre-BII stage to reach $86 \%$ of the cells (Fig. $6 \mathrm{~B}$, right panel).

In contrast, apoptosis is not detectable at the pre-BI stage (Fig. 6B, left panel), where a normal cell cycle is observed by BrdU incorporation (data not shown). A PCR assay was used to examine recombination of the flox alleles in these pre-BI cells. Indeed, flox alleles could be detected at a low level, suggesting that, in these cells, recombination was not complete (data not shown). Therefore, the normal cell cycle profile and lack of apoptosis observed in the remaining pre-BI cells is likely due to residual HDAC1 and/or HDAC2 protein. In other experiments, we found that one allele of HDAC1 or HDAC2 is sufficient to allow normal B-cell development

A

Large pre BII

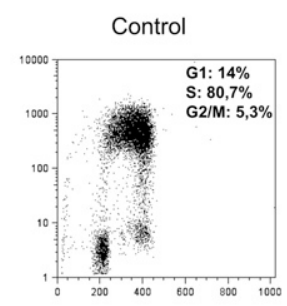

$H D A C 1 / 2^{F F}+C r e$
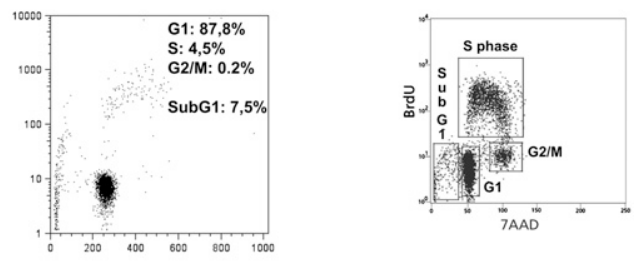

B
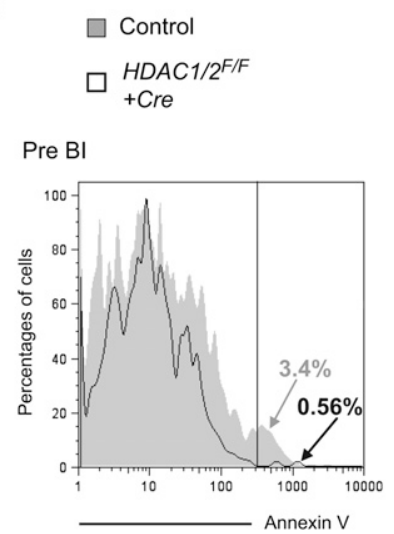

Large pre BII

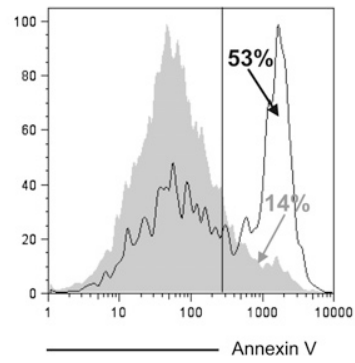

Small pre BII

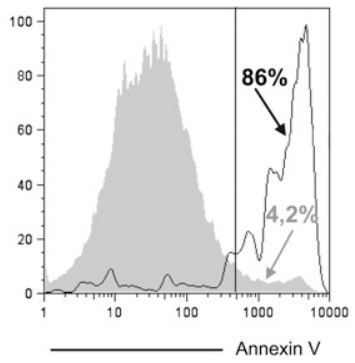

Figure 6. Ablation of $\mathrm{HDAC} 1$ and HDAC2 in vivo induces a severe cell cycle block in G1 and apoptosis. $(A)$ In vivo BrdU incorporation assay. Large pre-BII cells from $H D A C 1 / 2^{F / F}+m b 1$-cre or control $\left(H D A C 1 / 2^{F / F}\right)$ mice were sorted by FACS as indicated in the Materials and Methods. After fixation, permeabilization, and DNA digestion, the DNA was stained with an anti-BrdU antibody together with 7-AAD, and analysis was done by flow cytometry. Numbers in plots indicate the percentage of cells in each cell cycle phase. On the right, a schematic is presented in which the different cell populations are labeled. (B) Analysis of apoptosis by annexin $\mathrm{V}$ staining. Cells were stained for cell surface markers and annexin V, and were analyzed by flow cytometry. Data are representative of more than three mice of each genotype. 
(data not shown), similar to what has been observed in the case of cardiomyocyte development (Montgomery et al. 2007). The pre-BI cells that have fully deleted HDAC1 and HDAC2 are missing, similar to the pre-BII cells, but are too few to be detected efficiently by flow cytometry. In conclusion, in vivo deletion of HDAC1 and HDAC2 in the B-cell lineage leads to a very strong developmental block at the pre-BII stage, accompanied by arrest in G1 and high apoptosis, very similar to what was observed with MEFs in culture.

Nondividing $B$ cells do not require HDAC1 and HDAC2, but fail to proliferate in vitro

Results obtained in MEFs and early B-cell progenitors indicate that HDAC1 and HDAC2 have a critical function for cell cycle progression, suggesting the possibility that these proteins may be dispensable in resting cells. We tested this hypothesis in the B-cell paradigm and ablated HDAC1 and HDAC2 in nondividing terminally differentiated B cells. For this, cd23-cre mice (Kwon et al. 2008) were used, which induce deletion of floxed genes in immature and mature $\mathrm{B}$ cells, but not in early progenitors (see Fig. 5A). In vivo, the vast majority of mature B cells are nondividing and arrested in G1 until they were stimulated by foreign nonself antigens, in conjunction with a variety of cytokines and T-cell costimulation (for review, see McHeyzer-Williams and McHeyzer-Williams 2005). These cells can be cultured in vitro using different mitogenic stimuli. First, we analyzed splenic B cells of double-KO (HDAC1/2 $\left.2^{F / F}+c d 23-c r e\right)$ and control $\left(H D A C 1 / 2^{F / F}\right)$ mice by flow cytometry. As shown in Figure $7 \mathrm{~A}$, the percentages of $\mathrm{B}^{2} 20^{+} \mathrm{IgM}^{+}$mature B cells in the spleen were not affected by the deletion of HDAC1 and HDAC2. In addition, normal numbers of mature $\mathrm{B}$ cells were also obtained when additional mature B-cell markers, including CD21, CD23, and IgD, were used (data not shown). This indicates that HDAC1 and HDAC2 are dispensable in terminally differentiated and nondividing mature B cells. Next, we tested whether these mature $B$ cells could proliferate when stimulated in vitro. FACSpurified cells from double-KO or control mice were stained with the fluorescent dye carboxyfluorescein succinimidyl ester (CFSE) and cultured in vitro in the presence of lipopolysaccharide (LPS) and interleukin-4 (IL-4), after which analysis was done by flow cytometry. CFSE fluorescently stains the cells and, upon each cell division, the fluorescence intensity is halved; this allows us to accurately determine the number of divisions cells underwent (Weston and Parish 1990). As shown in Figure 7B, wild-type mature $\mathrm{B}$ cells divided about three to seven times within $72 \mathrm{~h}$, in contrast to zero or one division for double-KO B cells. These cells were also stained by annexin $\mathrm{V}$ to detect apoptosis induction; as shown in Figure 7C, massive induction of apoptosis was detected in double-KO B cells. Similar results were obtained when the cells were stimulated with anti-IgM Fab fragments (see Supplemental Fig. 7).

In conclusion, the deletion of HDAC1 and HDAC2 in naturally resting cells has no effect on viability until the

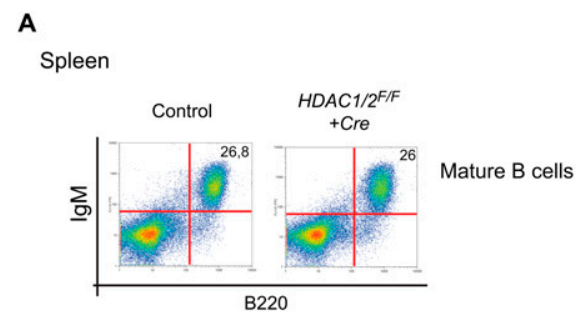

B
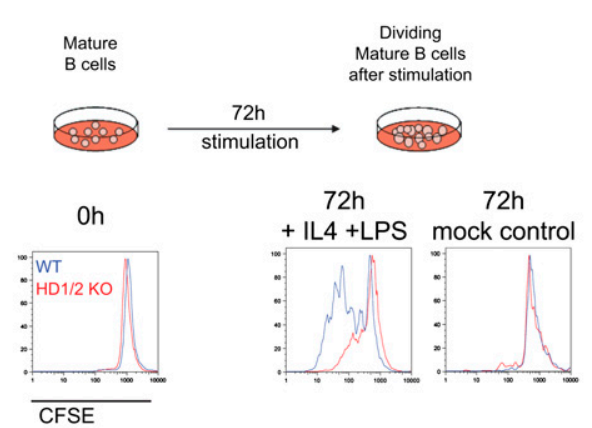

C

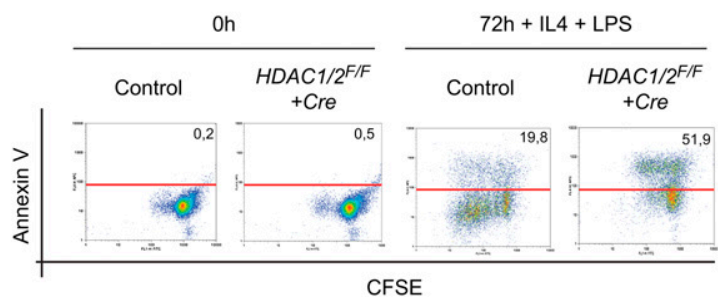

Figure 7. HDAC1 and HDAC2 are dispensable in mature resting $\mathrm{B}$ cells, but are required for their proliferation. $(A)$ Flow cytometric analysis of double-KO $\left(H D A C 1 / 2^{F / F}+C D 23-c r e\right)$ and control $\left(H D A C 1 / 2^{F / F}\right)$ mice. Single-cell suspensions from spleen were stained with the indicated antibody combination and analyzed by FACS. The dot plots presented are representative of an analysis done with four mice of each genotype. Numbers in plots represent the percentage of cells in the respective quadrant. (B) Mature B cells lacking HDAC1 and HDAC2 fail to proliferate in vitro. Splenic B cells were isolated from double$\mathrm{KO}$ or control mice, stained with CFSE, and induced to proliferate in vitro by addition of LPS + IL-4. The distribution of CFSE-positive cells was analyzed by FACS after $72 \mathrm{~h}$ of stimulation. $(C)$ Apoptosis induction in stimulated cells. Cells were stained with CFSE, stimulated for $72 \mathrm{~h}$ as above, and analyzed by annexin $\mathrm{V}$ staining.

cells are forced to re-enter the cell cycle. In this case, we observed a similar phenotype as in cycling cells: proliferation defect, followed by apoptosis.

\section{Discussion}

Using primary MEFs and B cells in vivo, we demonstrated the critical function of HDAC1 and HDAC2 in the cell cycle. Indeed, deletion of these two enzymes induces a strong proliferation block followed by apoptosis in both systems. A genome-wide transcription analysis using MEFs in which we can selectively inactivate HDAC1 and/or HDAC2 allowed us to focus on the molecular 
mechanisms of the cell cycle block. In cells where both enzymes are down-regulated, we observed, concomitant with a G1 arrest, a strong up-regulation of p21 and a weaker up-regulation of p57; in contrast, HDAC1 KO or HDAC2 Kd cells exhibit only partial deregulation of these proteins (Fig. 3C). ChIP experiments demonstrated that HDAC1 and HDAC2 both bind to the $p 21$ and $p 57$ gene promoters in wild-type cells (Fig. 4A), and we also showed that the mRNA level of these two genes is increased in the double-mutant MEFs (Fig. 4B). Taken together, these results demonstrate that, in wild-type cells, HDAC1 and HDAC2 directly repress the expression of the $p 21$ and $p 57$ genes. Thus, deletion of HDAC1 and HDAC2 releases this inhibition, leading to the accumulation of p21 and p57 proteins that stop the cell cycle in G1 by inhibiting the corresponding CDK complexes, mainly CDK2/cyclinE (for review, see Hunter and Pines 1994; Besson et al. 2008). Importantly, identical results were obtained when $\mathrm{HDACl} / 2 \mathrm{KO}$ cells were used (Supplemental Fig. 4).

The slow proliferation phenotype observed in HDAC1 $\mathrm{KO}$ cells is due to a partial release of the inhibition of the p21 gene promoter. Indeed, the proliferation defect in ES cells caused by the loss of HDAC1 (Lagger et al. 2002) can be restored fully by disruption of the $p 21$ gene (Zupkovitz et al. 2009|. Furthermore, we showed here that shRNAmediated down-regulation of p21 and p57 in MEFs deficient for $\mathrm{HDAC} 1$ and HDAC2 is sufficient to partially overcome the cell cycle block observed (Supplemental Fig. 6). Thereby, it is likely that a full rescue was not obtained because of incomplete efficacy of the shRNAs against $\mathrm{p} 21$ and $\mathrm{p} 57$.

We observed that p21 is up-regulated in HDAC1 KO cells but less than in double-mutant cells (Fig. 3C), suggesting an additive effect of HDAC2 deletion in $H D A C 1^{-1-}$ cells. This additive effect also suggests that a slow proliferation phenotype should be seen in HDAC2 Kd cells, unlike what we observed (Fig. 2A). A possible explanation may be due to the stronger up-regulation (threefold) of HDAC1 in HDAC2 Kd cells, compared with the weak $(\sim 1.4$-fold) up-regulation of HDAC2 in $H D A C 1^{-1-}$ cells (Fig. 1B). Obviously, the elevated expression of $p 21$ in HDAC1 KO cells indicates that the increase of HDAC2 at the $p 21$ promoter is not sufficient to compensate for the absence of HDAC1. In line with this, we observed that the level of p 21 and p 57 in HDAC2 Kd cells is reproducibly slightly lower than in wild-type cells, even though the mRNAs for $p 21$ and $p 57$ are not significantly lower in HDAC2 Kd cells than in wild type (Figs. 3C, 4B). The low expression levels of these genes in wild-type and HDAC2 Kd cells may preclude their accurate detection below a certain threshold. In addition, we cannot rule out that HDAC2 may also play a role in the protein stability of these CDKis. These observations imply that, in the absence of HDAC2, the increased HDAC1 protein level can repress the expression of these two genes more than in wild-type cells. Furthermore, it is likely that certain histone acetylation marks at these, and potentially other, promoters could be deacetylated only by HDAC1, but not by HDAC2, even when the amount of this enzyme is elevated on the promoter. Indeed, the $p 21$ and $p 57$ promoters are highly acetylated on $\mathrm{H} 4 \mathrm{~K} 8$ only in HDAC1 KO MEFs, suggesting that HDAC2 cannot deacetylate this residue on these promoters (Supplemental Fig. 8C). In addition, the loss of HDACl induces an increase of H3K14 acetylation that is not observed in the absence of HDAC2. The opposite is also true, as we see a specific effect of HDAC2 ablation on H4K12 acetylation. These findings indicate that HDAC1 and HDAC2 have clear target preferences; further experiments will allow us to dissect this specificity in greater detail.

These observations support the notion that HDAC1 and HDAC2 may have specific functions. Indeed, a recent study has shown that HDAC2 specifically regulates synaptic plasticity and memory formation, and that this cannot be compensated by overexpression of HDAC1 (Guan et al. 2009). Interestingly, Guan et al. (2009) have also noticed an effect of HDAC2 on H4K12 acetylation, as we observed in MEFs. Furthermore, a recent genomewide mapping of HDAC binding in human $\mathrm{T}$ cells has revealed that HDAC1 and HDAC2 do not entirely colocalize (Wang et al. 2009). In agreement with this, we found that between $40 \%$ and $60 \%$ of $\mathrm{HDAC} 1$ and $\mathrm{HDAC} 2$ are free of each other (Supplemental Fig. 8B). This suggests that only about half of HDAC1 and HDAC2 are present in the cell in a heterodimeric form associated with the known complexes, while the rest may be engaged in specific nonredundant functions. Furthermore, by coimmunoprecipitation experiments, we found that HDAC1 in the absence of HDAC2 is still able to interact with CoREST and Sin3a (this is also true vice versa), demonstrating that the integrity of known HDAC containing corepressor complexes Co-REST and Sin3a is not affected in the absence of one of these two enzymes (Supplemental Fig. 8A).

The specificity of these enzymes is also reflected in the genome-wide gene expression patterns, which are quite different between HDAC1 KO and HDAC2 Kd cells: About three times more genes were found deregulated in HDAC1 KO cells than in HDAC2 Kd cells (Supplemental Fig. 3), and only few genes were found to overlap $(17 \%)$. However, we cannot rule out that the HDAC2 Kd cells do not entirely mimic a full deletion of HDAC2. The gene ontology classification revealed that the first four groups of deregulated genes are the same in HDAC1 KO and HDAC2 Kd cells ("cellular movement," "cellular development," "cell growth and proliferation," and "cell death"), but in a different order (Fig. 2; data not shown).

Despite this weak similarity between the genome-wide expression profiles, genetic data in mice indicate that, in many cell types, $\mathrm{HDAC} 1$ and $\mathrm{HDAC} 2$ can be redundant, at least for some functions. Indeed, here we showed that B-cell differentiation occurs normally in the absence of HDAC1 or HDAC2, but that deletion of both induces a strong cell cycle block and apoptosis in early progenitors (Fig. 5C,D), leading to an almost complete absence of mature B cells. Experiments addressing the role of HDAC1 and HDAC2 in cardiomyocytes and neuronal precursors demonstrated that, in these systems as well, deletion of both enzymes is required to impair cellular 
differentiation, although the precise mechanism was not identified (Montgomery et al. 2007, 2009). Together, these results indicate that, in these different systems, HDAC1 and HDAC2 are redundant. In each of these cases, including the $\mathrm{B}$ cells reported here, deletion of HDAC1 and/or HDAC2 was induced in early lineage progenitors in which significant cellular proliferation takes place. There, the main function of HDAC1 and HDAC2 may be cell cycle control, for which these two proteins are largely redundant, but critical. Hence, the role of HDAC1 and HDAC2 in the cell cycle may obscure additional developmental roles these enzymes may have. In contrast, in terminally differentiated cells, like mature $\mathrm{B}$ cells, cellular proliferation is minimal, and other functions of HDAC1 or HDAC2 may be uncovered.

A study using human cancer cell lines (U2OS, MCF7, and MCF10A) showed, by a siRNA approach against HDAC1 and HDAC2, a similar phenotype, but weaker. Indeed, Senese et al. (2007) observed a proliferation defect and an enrichment of the cells in the G1 phase of the cell cycle. Yet, a complete proliferation block was not observed in two of the cell lines used, unlike what we described here for MEFs in culture, B cells in vivo, and what was described for cardiac and neuronal tissues (Montgomery et al. 2007, 2009). In the latter two studies, it was shown that one allele of either HDAC1 or HDAC2 is enough to maintain full cell viability. We obtained similar results in B cells, indicating that only one allele of HDAC1 or HDAC2 is sufficient to support normal B-cell development (data not shown). Thus, the difference in the cell cycle block observed in the different studies may reflect incomplete elimination of HDAC1 and/or HDAC2, leaving enough protein to sustain cell proliferation.

In addition to the partial block in G1, Senese et al. (2007) also observed apoptosis in G2/M. It seems likely that this difference is due to the use of a cancer cell line (U2OS). Indeed, a recent study has reported the deletion of HDAC1 and HDAC2 in MEFs and, in contrast to our study, has identified a cell cycle block in the $M$ phase, accompanied by multipolar spindle formation and mitotic catastrophes (Haberland et al. 2009). In this case, however, SV40 large T antigen-immortalized MEFs were used that were subsequently transformed with the mutant H-Ras V12G oncogene. Large T antigen, by binding $\mathrm{p} 53$, precludes activation of $\mathrm{p} 21$ and inhibits induction of apoptosis; furthermore, by also binding the Retinoblastoma protein $(\mathrm{Rb})$, large $\mathrm{T}$ circumvents the negative feedback of CDKis on $\mathrm{Rb}$ and effectively induces cell cycle entry (for review, see Ahuja et al. 2005). This activity of large $\mathrm{T}$ likely contributes to bypassing the G1 block we identified in double-deficient MEFs, and thus allows us to identify another stage where HDAC1 and HDAC2 act. Furthermore, in this study, a reduction in cell number was seen immediately upon inactivation of HDAC1 and HDAC2. In contrast, in our analysis of primary cells, following inactivation of HDAC1 and HDAC2, we first observed a cessation of cell proliferation for $>7$ d, but no apoptosis (Fig. 2A, Supplemental Fig. 4B; Haberland et al. 2009). Thus, in primary cells, up-regulation of p21 (and p57) triggered by the disappearance of
HDAC1 and HDAC2 may first induce mechanisms similar to a checkpoint response: The cells cease proliferation before eventually undergoing apoptosis.

In summary, we showed that the deletion of HDAC1 and HDAC2 induces p21 and p57 expression, leading to a cell cycle block in G1. The same experiment made in transformed cells has revealed other functions of HDAC1 and HDAC2 in mitosis that are not seen in primary cells (Haberland et al. 2009). This might be due to the early block in the cell cycle and/or specific features of the transformed cell that lack G1-phase regulation. In fact, it is well documented that the pathway involving p53 and p21 is often impaired in cancer cell lines (Weiss 2003; Soussi and Wiman 2007; Zambetti 2007). Moreover, we also observed that in vivo deletion of HDAC1 and HDAC2 in nondividing mature B cells that are quiescent and arrested in G1 has no effect on their viability; however, when these cells are cultured, they fail to proliferate, and undergo rapid apoptosis (Fig. 7). Taken together, these results suggest that HDAC1 and HDAC2 function at several distinct stages of the cell cycle, and absence of these two enzymes has little negative effect on resting primary cells. Therefore, inhibitors specific for HDAC1 and HDAC2 would target specifically proliferating cancer cells-potentially even in the context of a p53 mutation-and would be valuable candidates for chemotherapy.

\section{Materials and methods}

Flow cytometry (FACS)

Cell staining was done according to standard procedures. Flow cytometry analysis was performed on a FACSCalibur (BD Biosciences) by gating on live cells. Samples were analyzed by using Flow-Jo (Tree Star) software. Cell sorting was performed on a MoFlo (DakoCytomation). The purity of sorted cells was checked by reanalysis.

The following mAbs were from BD Biosciences: RA3-6B2 (anti-B220) conjugated to allophycocyanin (APC), ACK45 (antic-kit) conjugated to phycoerythrin (PE) or biotinylated, S7 (antiCD43) conjugated to fluorescein isothiocyanate (FITC), 1D3 (anti-CD19) conjugated to APC-Cy7 and 7D4 (anti-CD25) biotinylated. 1B4B1 (anti-IgM) conjugated to FITC was from Southern Biotech. 6D5 (anti-CD19) conjugated to PE was from Biolegend. Streptavidin conjugated to PE-Cy5.5 was from CALTAG. For the annexin $\mathrm{V}$ staining, we followed the manufacturer's protocol from BD Biosciences.

\section{BrdU incorporation assay}

For in vivo incorporation, mice were injected intraperitoneally with $20 \mu \mathrm{g}$ of $\mathrm{BrdU}$ and were sacrificed $30 \mathrm{~min}$ later. Bone marrow single-cell suspensions were prepared, stained, and sorted with the combination of antibodies against the following cell surface molecules: $\mathrm{CD} 19^{+}, \mathrm{CD}_{4} 3^{+}$, and $\mathrm{CD} 25^{-}$. BrdU staining using sorted cells was performed according to the manufacturer's protocol of BrdU flow kit (BD Bioscience). Analysis was performed on a FACSCalibur and analyzed by Flow-Jo software.

For cells in culture, a 30-min pulse of BrdU was done, followed by the staining procedure based on the manufacturer's instructions (BD Bioscience). 
Virus production and MEF infections

Lipofectamine 2000 was used to transfect $5 \times 10^{6}$ Ecophenix packaging cell line in a $100-\mathrm{mm}$ dish with $24 \mu \mathrm{g}$ of plasmid (pMSCV_CreERT2_puromycin, pMSCV_CreERT2_eGFP, or pMSCV_shRNA_puromycin). Fresh medium was added $5 \mathrm{~h}$ later, and the virus supernatant was collected after 60 -h incubation at $32^{\circ} \mathrm{C}$, filtrated on $0.45-\mu \mathrm{m}$ filters, and supplemented with $5 \mu \mathrm{g} / \mathrm{mL}$ polybrene. The sequence of the miRNAs is indicated in Supplemental Table 2.

MEF cells $\left(5 \times 10^{5}\right)$ were seeded on a $100-\mathrm{mm}$ dish the day before the infection. Five microliters of virus supernatant were used per 100-mm dish. The cells were spun at $1000 \mathrm{~g}$ for $1 \mathrm{~h}$ and then incubated for $5 \mathrm{~h}$ at $37^{\circ} \mathrm{C}$ before changing the medium. When eGFP was used as a marker, the cells were sorted on a MoFlo (DakoCytomation). When puromycin was used as a marker, after $72 \mathrm{~h}$ of expression, the cells were selected in the presence of $1-1.5 \mu \mathrm{g} / \mathrm{mL}$ during $48 \mathrm{~h}$.

\section{Protein extracts and Western blot}

Cells were trypsinized, washed with cold phosphate-buffered saline (PBS), and either snap-frozen in liquid nitrogen or lysed immediately in buffer $(50 \mathrm{mM}$ Hepes at $\mathrm{pH} 7.5,300 \mathrm{mM} \mathrm{NaCl}$, $25 \mathrm{mM}$ glycerophosphate, $25 \mathrm{mM} \mathrm{NaF}, 5 \mathrm{mM}$ EGTA, $1 \mathrm{mM}$ EDTA, $0.5 \%$ NP40, $0.05 \%$ NaDoc) supplemented with $1 \mathrm{mM}$ phenylmethylsulfonyl fluoride and the Complete protease inhibitor cocktail (Roche). Lysates were centrifuged for $10 \mathrm{~min}$ at $4^{\circ} \mathrm{C}$, and supernatants were resolved by electrophoresis on $10 \%$ or $15 \%$ SDS-PAGE gels and subsequently blotted to nitrocellulose membranes (Protran) when ECL (Amersham) was used and PVDF (Millipore) when the Odyssey system (Li-Cor) was used. The antibodies used in this study were the following: HDAC1 and HDAC2 (gift from Christian Seiser, Vienna Biocenter), actin (Ab-5; Neo Markers), cyclin D2 (MBL International Corporation), cyclin B1 (MBL International Corporation), p15 (Cell Signaling), p16 (M-156, Santa Cruz Biotechnologies), p21 (F-5, Santa Cruz Biotechnologies), p27 (F-8, Santa Cruz Biotechnologies), p57 (p2735, Sigma), Co-REST (07-455, Millipore), Sin3a (06-913, Millipore), acetyl H4 (06-866, Millipore), acetyl H3 (06-599, Millipore), acetyl H3K14 (07-353, Millipore), acetyl H4K5 (07327, Millipore), acetyl H4K8 (07-328, Millipore), and acetyl H4K12 (06-761, Millipore).

\section{Proteins immunoprecipitation experiments}

Protein extracts $(250 \mu \mathrm{g})$ were used for immunoprecipitation with either HDAC1 or HDAC2 antibody. Twenty microliters of antibodies were incubated with protein extracts for $4 \mathrm{~h}$ at $4^{\circ} \mathrm{C}$ on a rotating wheel. Then, $20 \mu \mathrm{L}$ of protein $\mathrm{G}$ sepharose $4 \mathrm{FF}$ (GE Healthcare) were added for $1 \mathrm{~h}$ at $4^{\circ} \mathrm{C}$. The beads were washed three times with lysis buffer supplemented with $250 \mathrm{mM} \mathrm{NaCl}$. Finally, the beads were boiled in SDS loading buffer for $10 \mathrm{~min}$.

\section{ChIP experiments}

ChIP assays were performed with a $150-\mathrm{mm}$ dish $80 \%$ confluent as described on the Abcam Web site, with minor modifications. DNA was sheared with a sonicator to an average fragment size of 500 base pairs. Each sample was split into two to perform immunoprecipitation with either HDAC1 or HDAC2 antibodies. Dynabeads (Invitrogen) were used instead of protein G beads. PCR was performed using the MESA GREEN qPCR Mastermix for SYBR Green (Eurogentec) on ABI PRYSM 7000 cycler. Each reported $\mathrm{ChIP}$ value is an average of three independent experiments $( \pm \mathrm{SD})$ with the PCR amplification performed in triplicate. Values are normalized to background levels detected with probes for a control locus (actin). Primer sequences are indicated in Supplemental Table 2.

\section{$q R T-P C R$}

RNA was purified with the RNeasy Minikit (Qiagen) according to the manufacturer's protocol. cDNA was synthesized by using the Thermoscript Reverse Transcriptase Kit (Invitrogen). Oligo $\mathrm{dT}$ primers provided in the kit were used for the first strand synthesis. qPCR was performed on an ABI PRISM 7000 Sequence Detection System (Applied Biosystems) using a MESA GREEN qPCR MasterMix Plus for SYBR Assay (Eurogentec). The amount of $p 21$ and $p 57$ transcripts was normalized by Hprt1 as follow: Relative expression levels were calculated using the formula $2^{-(\Delta \mathrm{Ct})}$, where $\Delta \mathrm{Ct}$ is $\mathrm{Ct}_{\text {(gene of interest) }}-\mathrm{Ct}_{(\text {Hprt1) }}$ and $\mathrm{Ct}$ is the cycle at which the threshold is crossed. Primers used for the PCR detection are indicated in Supplemental Table 2.

\section{Mature B-cell isolation and in vitro CFSE proliferation assays}

Mature B cells were sorted with the combination of antibodies against the following cell surface molecules: $\mathrm{CD} 21^{+}, \mathrm{CD}_{2} 3^{+}$, $\mathrm{B}^{2} 20^{+}$, and human $\mathrm{CD}^{+}$. Sorted cells were stained with $10 \mu \mathrm{M}$ CFSE in prewarmed RPMI- 1640 medium for $10 \mathrm{~min}$ at $37^{\circ} \mathrm{C}$. The reaction was stopped by adding an equal volume of FBS, and then cells were washed thoroughly twice with RPMI-1640 medium containing $10 \%$ FBS. Cell numbers were counted by using a ViCell XR counter, and the same numbers of cells were plated with $1 \times 10^{6}$ cells per milliliter concentration. B-cell cultures were carried out with or without mitogens; either LPS $(10 \mu \mathrm{g} / \mathrm{mL})$ and IL-4 $(10 \mathrm{ng} / \mathrm{mL})$ or anti-IgM Fab fragments $(25 \mu \mathrm{g} / \mathrm{mL})$ in RPMI1640 medium containing 10\% FBS, $2 \mathrm{mM}$ L-glutamine, $1 \mathrm{mM}$ Na-pyruvate, and $50 \mu \mathrm{M}$ 2-mercaptoethanol. After 72 h, CFSE and annexin $\mathrm{V}$ staining were visualized by flow cytometry.

\section{Acknowledgments}

We thank Michael Reth (Max-Planck Institute of Immunobiology, Freiburg, Germany) for generously providing mb1-cre transgenic mice, Meinrad Busslinger (Research Institute of Molecular Pathology, Vienna, Austria) for kindly sharing cd23-cre transgenic mice, Edward Seto (H. Lee Moffitt Cancer Center and Research Institute, Tampa, FL) for the mouse HDAC2 genomic construct, Antoine Peters (Friedrich Miescher Institute for Biomedical Research, Basel, Switzerland) for antibodies and useful discussions, and Ton Rolink (Basel University), Nancy Hynes (FMI, Basel), and Saadi Khochbin (Institute Albert Bonniot, Grenoble, France) for suggestions and comments on the manuscript. We also thank Patrick Kopp and Jean-François Spetz for excellent ES cell work and generation of KO mice, Camille Du Roure for discussions and help with FACS sorting, Gabriele Matthias and Chun Cao for excellent assistance and discussions, and all members of the Matthias laboratory for useful discussions and suggestions. This work was supported by the Novartis Research Foundation, the Swiss Cancer League/Oncosuisse (grant OCS-02177-02-2008) and the Austrian Science Fund (grant P16443).

\section{References}

Ahuja D, Saenz-Robles MT, Pipas JM. 2005. SV40 large T antigen targets multiple cellular pathways to elicit cellular transformation. Oncogene 24: 7729-7745.

Allfrey VG, Faulkner R, Mirsky AE. 1964. Acetylation and methylation of histones and their possible role in the regulation of Rna synthesis. Proc Natl Acad Sci 51: 786-794. 
Bali P, Pranpat M, Bradner J, Balasis M, Fiskus W, Guo F, Rocha K, Kumaraswamy S, Boyapalle S, Atadja P, et al. 2005. Inhibition of histone deacetylase 6 acetylates and disrupts the chaperone function of heat shock protein 90: A novel basis for antileukemia activity of histone deacetylase inhibitors. J Biol Chem 280: 26729-26734.

Berger SL. 2007. The complex language of chromatin regulation during transcription. Nature 447: 407-412.

Besson A, Dowdy SF, Roberts JM. 2008. CDK inhibitors: Cell cycle regulators and beyond. Dev Cell 14: 159-169.

Guan JS, Haggarty SJ, Giacometti E, Dannenberg JH, Joseph N, Gao J, Nieland TJ, Zhou Y, Wang X, Mazitschek R, et al. 2009. HDAC2 negatively regulates memory formation and synaptic plasticity. Nature 459: 55-60.

Haberland M, Johnson A, Mokalled MH, Montgomery RL, Olson EN. 2009. Genetic dissection of histone deacetylase requirement in tumor cells. Proc Natl Acad Sci 106: 7751-7755.

Hobeika E, Thiemann S, Storch B, Jumaa H, Nielsen PJ, Pelanda $\mathrm{R}$, Reth M. 2006. Testing gene function early in the B cell lineage in mb1-cre mice. Proc Natl Acad Sci 103: 13789-13794.

Hubbert C, Guardiola A, Shao R, Kawaguchi Y, Ito A, Nixon A, Yoshida M, Wang XF, Yao TP. 2002. HDAC6 is a microtubule-associated deacetylase. Nature 417: 455-458.

Hunter T, Pines J. 1994. Cyclins and cancer. II: Cyclin D and CDK inhibitors come of age. Cell 79: 573-582.

Indra AK, Warot X, Brocard J, Bornert JM, Xiao JH, Chambon $\mathrm{P}$, Metzger D. 1999. Temporally-controlled site-specific mutagenesis in the basal layer of the epidermis: Comparison of the recombinase activity of the tamoxifen-inducible CreER(T) and Cre-ER(T2) recombinases. Nucleic Acids Res 27: $4324-4327$.

Kovacs JJ, Murphy PJ, Gaillard S, Zhao X, Wu JT, Nicchitta CV, Yoshida M, Toft DO, Pratt WB, Yao TP. 2005. HDAC6 regulates Hsp90 acetylation and chaperone-dependent activation of glucocorticoid receptor. Mol Cell 18: 601-607.

Kurdistani SK, Robyr D, Tavazoie S, Grunstein M. 2002. Genome-wide binding map of the histone deacetylase Rpd3 in yeast. Nat Genet 31: 248-254.

Kurdistani SK, Tavazoie S, Grunstein M. 2004. Mapping global histone acetylation patterns to gene expression. Cell 117: 721-733.

Kwon K, Hutter C, Sun Q, Bilic I, Cobaleda C, Malin S, Busslinger M. 2008. Instructive role of the transcription factor E2A in early B lymphopoiesis and germinal center B cell development. Immunity 28: 751-762.

Lagger G, O'Carroll D, Rembold M, Khier H, Tischler J, Weitzer G, Schuettengruber B, Hauser C, Brunmeir R, Jenuwein T, et al. 2002. Essential function of histone deacetylase 1 in proliferation control and CDK inhibitor repression. EMBO $J$ 21: 2672-2681.

Lagger G, Doetzlhofer A, Schuettengruber B, Haidweger E, Simboeck E, Tischler J, Chiocca S, Suske G, Rotheneder H, Wintersberger E, et al. 2003. The tumor suppressor p53 and histone deacetylase 1 are antagonistic regulators of the cyclin-dependent kinase inhibitor p21/WAF1/CIP1 gene. Mol Cell Biol 23: 2669-2679.

Lin YC, Lin JH, Chou CW, Chang YF, Yeh SH, Chen CC. 2008. Statins increase p21 through inhibition of histone deacetylase activity and release of promoter-associated HDAC1/2. Cancer Res 68: 2375-2383.

Luo J, Su F, Chen D, Shiloh A, Gu W. 2000. Deacetylation of p53 modulates its effect on cell growth and apoptosis. Nature 408: 377-381.

Malumbres M, Ortega S, Barbacid M. 2000. Genetic analysis of mammalian cyclin-dependent kinases and their inhibitors. Biol Chem 381: 827-838.
Matthias P, Rolink AG. 2005. Transcriptional networks in developing and mature B cells. Nat Rev Immunol 5: 497508.

McHeyzer-Williams LJ, McHeyzer-Williams MG. 2005. Antigenspecific memory B cell development. Annu Rev Immunol 23: 487-513.

Minucci S, Pelicci PG. 2006. Histone deacetylase inhibitors and the promise of epigenetic (and more) treatments for cancer. Nat Rev Cancer 6: 38-51.

Montgomery RL, Davis CA, Potthoff MJ, Haberland M, Fielitz J, Qi X, Hill JA, Richardson JA, Olson EN. 2007. Histone deacetylases 1 and 2 redundantly regulate cardiac morphogenesis, growth, and contractility. Genes \& Dev 21: 17901802.

Montgomery RL, Hsieh J, Barbosa AC, Richardson JA, Olson EN. 2009. Histone deacetylases 1 and 2 control the progression of neural precursors to neurons during brain development. Proc Natl Acad Sci 106: 7876-7881.

Roh TY, Cuddapah S, Zhao K. 2005. Active chromatin domains are defined by acetylation islands revealed by genome-wide mapping. Genes \& Dev 19: 542-552.

Rolink A, Haasner D, Nishikawa S, Melchers F. 1993. Changes in frequencies of clonable pre B cells during life in different lymphoid organs of mice. Blood 81: 2290-2300.

Rountree MR, Bachman KE, Baylin SB. 2000. DNMT1 binds HDAC2 and a new co-repressor, DMAP1, to form a complex at replication foci. Nat Genet 25: 269-277.

Senese S, Zaragoza K, Minardi S, Muradore I, Ronzoni S, Passafaro A, Bernard L, Draetta GF, Alcalay M, Seiser C, et al. 2007. Role for histone deacetylase 1 in human tumor cell proliferation. Mol Cell Biol 27: 4784-4795.

Soussi T, Wiman KG. 2007. Shaping genetic alterations in human cancer: The p53 mutation paradigm. Cancer Cell 12: 303-312.

Taplick J, Kurtev V, Kroboth K, Posch M, Lechner T, Seiser C. 2001. Homo-oligomerisation and nuclear localisation of mouse histone deacetylase 1. J Mol Biol 308: 27-38.

Trivedi CM, Luo Y, Yin Z, Zhang M, Zhu W, Wang T, Floss T, Goettlicher M, Noppinger PR, Wurst W, et al. 2007. Hdac2 regulates the cardiac hypertrophic response by modulating Gsk3 $\beta$ activity. Nat Med 13: 324-331.

Vogelauer M, Wu J, Suka N, Grunstein M. 2000. Global histone acetylation and deacetylation in yeast. Nature 408: 495498.

Wang Z, Zang C, Cui K, Schones DE, Barski A, Peng W, Zhao K. 2009. Genome-wide mapping of HATs and HDACs reveals distinct functions in active and inactive genes. Cell 138: 1019-1031.

Weiss RH. 2003. p21Waf1/Cip1 as a therapeutic target in breast and other cancers. Cancer Cell 4: 425-429.

Weston SA, Parish CR. 1990. New fluorescent dyes for lymphocyte migration studies. Analysis by flow cytometry and fluorescence microscopy. I Immunol Methods 133: 87-97.

Yang XI, Seto E. 2008. The Rpd3/Hda1 family of lysine deacetylases: From bacteria and yeast to mice and men. Nat Rev Mol Cell Biol 9: 206-218.

Zambetti GP. 2007. The p53 mutation 'gradient effect' and its clinical implications. I Cell Physiol 213: 370-373.

Zhang Y, Li N, Caron C, Matthias G, Hess D, Khochbin S, Matthias P. 2003. HDAC-6 interacts with and deacetylates tubulin and microtubules in vivo. EMBO I 22: 1168-1179.

Zhang Y, Kwon S, Yamaguchi T, Cubizolles F, Rousseaux S, Kneissel M, Cao C, Li N, Cheng HL, Chua K, et al. 2008. Mice lacking histone deacetylase 6 have hyperacetylated tubulin but are viable and develop normally. Mol Cell Biol 28: $1688-1701$. 
Zimmermann S, Kiefer F, Prudenziati M, Spiller C, Hansen J, Floss T, Wurst W, Minucci S, Gottlicher M. 2007. Reduced body size and decreased intestinal tumor rates in HDAC2mutant mice. Cancer Res 67: 9047-9054.

Zupkovitz G, Tischler J, Posch M, Sadzak I, Ramsauer K, Egger G, Grausenburger R, Schweifer N, Chiocca S, Decker T, et al. 2006. Negative and positive regulation of gene expression by mouse histone deacetylase 1. Mol Cell Biol 26: 7913-7928.

Zupkovitz G, Grausenburger R, Brunmeir R, Senese S, Tischler J, Jurkin J, Rembold M, Meunier D, Egger G, Lagger S, et al. 2009. The cyclin dependent kinase inhibitor p21 is a crucial target for Histone deacetylase 1 as regulator of cellular proliferation. Mol Cell Biol. doi: 10.1128/MCB.01500-09. 


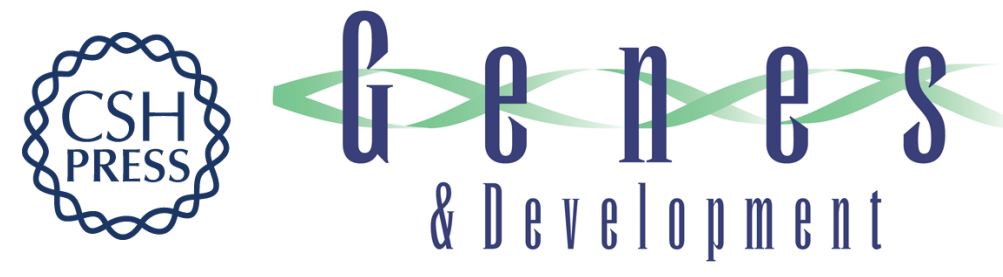

\section{Histone deacetylases 1 and 2 act in concert to promote the G1-to-S progression}

Teppei Yamaguchi, Fabien Cubizolles, Yu Zhang, et al.

Genes Dev. 2010, 24:

Access the most recent version at doi:10.1101/gad.552310

Supplemental
Material http://genesdev.cshlp.org/content/suppl/2010/02/18/24.5.455.DC1

References This article cites 43 articles, 17 of which can be accessed free at: http://genesdev.cshlp.org/content/24/5/455.full.html\#ref-list-1

License

Email Alerting

Receive free email alerts when new articles cite this article - sign up in the box at the top Service right corner of the article or click here.

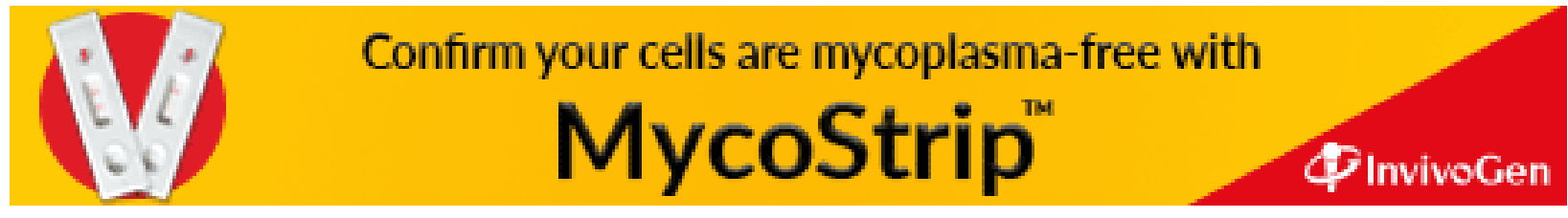

